\title{
On Applications of Fractional Calculus Involving Summations of Series
}

\author{
Praveen Agarwal \\ Department of Mathematic Anand International College of Engineer ing Jaipur, 302012, India
}

\begin{abstract}
A significantly large number of earlier works on the subject of fractional calculus give interesting account of the theory and applications of fractional calculus operators in many different areas of mathematical analysis (such as ordinary and partial differential equations, integral equations, special functions, summation of series, et cetera). The ma in object of the present paper is to obtain number of summations of series concerning generalized hypergeometric functions. Our finding provides interesting unifications and extensions of a number of new and known results.
\end{abstract}

Keywords Fractional Calculus, Special Function, Summation of Series, Generalized Leibniz Rule, Generalized Hypergeometric Series, Laguerre Polynomials

\section{Introduction}

One of the most frequently encountered tools in the theory of fractional calculus (that is, differentiation and integration of an arbitrary real or complex order) is furn ished by the familiar differintegral operator ${ }_{a} D_{z}^{\alpha}$ defined and represented by Oldham and Spanier[12]:

$$
\left.{ }_{a} D_{z}^{\alpha} f(z)=\frac{1}{\Gamma(-\alpha)} \int_{a}^{z}(z-y)^{-\alpha-1} f(y) d y\right)
$$

and

$$
\begin{aligned}
{ }_{a} D_{z}^{\alpha} f(z) & =D_{z}^{n}\left[\frac{1}{\Gamma(n-\alpha)} \int_{a}^{z}(z-y)^{n-\alpha-1} f(y) d y\right] \\
& =D_{z}^{n}\left[a D_{z}^{\alpha-n} f(z)\right], \quad \mathfrak{R}(\alpha) \geq 0 .
\end{aligned}
$$

where $\mathrm{n}$ is the least positive integer such that $\mathrm{n}>\mathrm{q}$.

The operator ${ }_{a} D_{z}^{\alpha}$ provides a generalization of the familiar differential and integral operator, viz., $D\left(\equiv \frac{d}{d z}\right)$ and $\left.D^{-1}\right\}$.

For a $=0$ the operator $D_{z}^{\alpha}$ is given by

$$
D_{z}^{\alpha}={ }_{0} D_{z}^{\alpha}(\alpha \in \mathbb{C})
$$

* Corresponding author:

goyal.praveen2000@gmail.com (Praveen Agarwal)

Published online at http://journal.sapub.org/ajms

Copyright (C) 2012 Scientific \& Academic Publishing. All Rights Reserved corresponding ess entially to the classical Riemann-Liouville fractional derivative (or integral) of order $\alpha$ (or $-\alpha$ ). Moreover, when $a \rightarrow \infty$, Equation (1.1) may be identified with the definition of the familiar Weyl fractional derivative (or integral) of order $\alpha$ (or $-\alpha$ ).

In recent years there has appeared a great deal of literature discussing the application of the aforementioned fractional calculus operators in a number of areas of mathematical analys is (such as ordinary and partial differential equations, integral equations, summation of series, et cetera) and now stands on fairly firm footing through the research contribution of various authors (cf., e.g.,[2],[5-7],[9-14],[16] and[17]). In the present paper main object is to obtain number of summations of series concerning generalized hypergeometric functions.

The familiar Leibniz rule for ord inary derivatives admits itself of the following extension in terms of the Riemann-Liouville operator $D_{z}^{\alpha}$ defined by (1.3):

$$
\begin{aligned}
& D_{z}^{\alpha}[u(z) v(z)]=\sum_{n=0}^{\infty}\left(\begin{array}{l}
\alpha \\
n
\end{array}\right) \\
& D_{z}^{\alpha-n}[u(z)] D_{z}^{n}[v(z)](\alpha \in \mathbb{C})
\end{aligned}
$$

The generalized Leibniz rule (1.4), wh ich was also applied earlier by Galué et al.[5] order to derive the summation identity:

$$
\begin{gathered}
\sum_{n=1}^{\infty}(-1)^{n-1} \frac{(\alpha)_{n}}{n !} \sum_{k=0}^{\infty}\left(\begin{array}{l}
\alpha \\
k
\end{array}\right) D_{z}^{-k-n}[u(z)] D_{z}^{n+k}[v(z)] \\
=\sum_{n=1}^{\infty}\left(\begin{array}{l}
\alpha \\
n
\end{array}\right) D_{z}^{-n}[u(z)] D_{z}^{n}[v(z)](\alpha \in \mathbb{C})
\end{gathered}
$$

Suffers from an apparent drawback in the sence that the interchange of the function $\mathrm{u}(\mathrm{z})$ and $\mathrm{v}(\mathrm{z})$ on the right-hand 
side is not obvious. (see also Galué et al. for several symmetrical generalized of (1.4) considered by Watanabe[17] summation formu las [6] contained in the Chen-Srivastava[2] ) and Osler[13], without such a drawback, is given by (cf., e.g., which she deduced by suitable specializing the function $\mathrm{u}(\mathrm{z})$ and $\mathrm{v}(\mathrm{z})$ in the summation identity (1.5) above.) A further

Samko et a1.[14, p. 316, Equation (17.12)]):

$$
D_{z}^{\alpha}[u(z) v(z)]=\sum_{n=-\infty}^{\infty}\left(\begin{array}{l}
\alpha \\
\eta+n
\end{array}\right) D_{z}^{\alpha-\eta-n}[u(z)] D_{z}^{\eta+n}[v(z)] \quad(\alpha, \eta \in \mathbb{C})
$$

which, in the special case when $(\eta=0)$,yields the Leibniz rule (1.4).

The condition of valid ity of the above results is given by T. J. Osler[13, p. 664-665]).

The generalized hypergeometric function of one variable viz., ${ }_{p} F_{q}[. ; ; ; z]$ defined and represented as follows (see e.g.[15, p.19]) is also required here:

$$
{ }_{p} F_{q}\left[\begin{array}{l}
\left(a_{p}\right) ; \\
\left(b_{q}\right) ;
\end{array}\right]=\sum_{n=0}^{\infty} \frac{\prod_{j=1}^{p}\left(a_{j}\right)_{n}}{\prod_{j=1}^{q}\left(b_{j}\right)_{n}} \frac{z^{n}}{n !} ; \text { provided } \quad p \leq q \quad \text { or } \quad p=q+1 \text { and } \quad|z|<1
$$

The Laguerre polynomials defined and represented as follows (see e.g.[1, p.775]):

$$
L_{n}^{a}(x)=\frac{(\alpha+1)_{n}}{n !}{ }_{1} F_{1}(-n ; \alpha+1 ; x)
$$

where $(\alpha)_{n}$ is the Pochhammer symbol and ${ }_{1} F_{1}[a ; b ; x]$ is a confluent hypergeometric function of the first kind (see e.g.[8]).

\section{Main results}

In this section, we shall establish some new summation formu lae for the generalized hypergeometric function ${ }_{p} F_{q}[$.$] .$

Summation For mulae 2.1

$$
\begin{aligned}
& \sum_{n=-\infty}^{\infty}\left(\begin{array}{c}
\alpha \\
\eta+n
\end{array}\right)\left[\frac{\left[F_{1}(\mu ; \mu-\alpha+\eta+n ; i a z)+{ }_{1} F_{1}(\mu ; \mu-\alpha+\eta+n ;-i a z)\right]}{\Gamma(\lambda-\eta-n) \Gamma(\mu-\alpha+\eta+n)}\right. \\
& \quad\left[{ }_{1} F_{1}(\lambda ; \lambda-\eta-n ; i b z)+{ }_{1} F_{1}(\lambda ; \lambda-\eta-n ;-i b z)\right] \\
& =\frac{\Gamma(\mu+\lambda-1)}{\Gamma(\mu) \Gamma(\lambda) \Gamma(\mu+\lambda-\alpha-1)}\left[{ }_{1} F_{1}(\mu+\lambda-1 ; \mu+\lambda-\alpha-1 ; i(a+b) z)+{ }_{1} F_{1}(\mu+\lambda-1 ; \mu+\lambda-\alpha-1 ;-i(a+b) z)\right. \\
& \left.\quad+{ }_{1} F_{1}(\mu+\lambda-1 ; \mu+\lambda-\alpha-1 ; i(a-b) z)+{ }_{1} F_{1}(\mu+\lambda-1 ; \mu+\lambda-\alpha-1 ;-i(a-b) z)\right]
\end{aligned}
$$

where $\mathfrak{R}(\lambda+\mu)>1, \mathfrak{R}(\lambda)>0$, and $\mathfrak{R}(\mu)>0$.

The conditions of validity of the above results follow easily from the conditions given by T. J. Osler[13, p. 664-665]).

Summation For mulae 2.2

$$
\begin{aligned}
& \sum_{n=-\infty}^{\infty}\left(\begin{array}{c}
\alpha \\
\eta+n
\end{array}\right) \frac{\left[{ }_{1} F_{1}(\mu ; \mu-\alpha+\eta+n ; i a z)-{ }_{1} F_{1}(\mu ; \mu-\alpha+\eta+n ;-i a z)\right]}{\Gamma(\lambda-\eta-n) \Gamma(\mu-\alpha+\eta+n)} \\
& \quad\left[{ }_{1} F_{1}(\lambda ; \lambda-\eta-n ; i b z)+{ }_{1} F_{1}(\lambda ; \lambda-\eta-n ;-i b z)\right] \\
& =\frac{(2 i) \Gamma(\mu+\lambda-1)}{\Gamma(\mu) \Gamma(\lambda) \Gamma(\mu+\lambda-\alpha-1)}\left[{ }_{1} F_{1}(\mu+\lambda-1 ; \mu+\lambda-\alpha-1 ; i(a+b) z)-{ }_{1} F_{1}(\mu+\lambda-1 ; \mu+\lambda-\alpha-1 ;-i(a+b) z)\right. \\
& \left.\quad+{ }_{1} F_{1}(\mu+\lambda-1 ; \mu+\lambda-\alpha-1 ; i(a-b) z)-{ }_{1} F_{1}(\mu+\lambda-1 ; \mu+\lambda-\alpha-1 ;-i(a-b) z)\right]
\end{aligned}
$$

provided that

$$
\mathfrak{R}(\lambda+\mu)>1, \mathfrak{R}(\lambda)>0 \text {, and } \mathfrak{R}(\mu)>0 .
$$


The conditions of validity of the above results follow easily from the conditions given by T. J. Osler[13, p. 664-665]).

Summation Formulae 2.3

$$
\begin{aligned}
& \sum_{n=-\infty}^{\infty}\left(\begin{array}{c}
\alpha \\
\eta+n
\end{array}\right) \frac{{ }_{2} F_{2}\left(a+m+1, \mu ; a+1, \mu-\alpha+\eta+n ;-k_{1} z\right)}{\Gamma(\lambda-\eta-n) \Gamma(\mu-\alpha+\eta+n)}{ }_{1} F_{1}\left(\lambda ; \lambda-\eta-n ;-k_{2} z\right) \\
= & \frac{\Gamma(\mu+\lambda-1)}{\Gamma(\mu) \Gamma(\lambda) \Gamma(\mu+\lambda-\alpha-1)}{ }_{2} F_{2}\left(a+m+1, \mu+\lambda-1 ; a+1, \mu+\lambda-\alpha-1 ;-\left(k_{1}+k_{2}\right) z\right)
\end{aligned}
$$

where $\mathfrak{R}(\lambda+\mu)>1, \mathfrak{R}(\lambda)>0$, and $\mathfrak{R}(\mu)>0$.

The conditions of validity of the above results follow easily from the conditions given by T. J. Osler[13, p. 664-665]).

Summati on For mulae 2.4

$$
\begin{aligned}
& \sum_{n=-\infty}^{\infty}\left(\begin{array}{c}
\alpha \\
\eta+n
\end{array}\right) \frac{{ }_{1} F_{1}\left(\mu ; \frac{1}{2}, \mu-\alpha+\eta+n ; \frac{-a^{2} z}{4}\right)}{\Gamma(\lambda-\eta-n) \Gamma(\mu-\alpha+\eta+n)}{ }_{1} F_{1}\left(\lambda ; \frac{1}{2}, \lambda-\eta-n ; \frac{-b^{2} z}{4}\right) \\
& =\frac{\Gamma(\mu+\lambda-1)}{\Gamma(\mu+\lambda-\alpha-1)}\left[{ }_{1} F_{1}\left(\mu+\lambda-1 ; \frac{1}{2}, \mu+\lambda-\alpha-1 ;-\frac{(a+b)^{2} z}{4}\right)\right. \\
& \left.\quad+{ }_{1} F_{1}\left(\mu+\lambda-1 ; \frac{1}{2}, \mu+\lambda-\alpha-1 ;-\frac{(a-b)^{2} z}{4}\right)\right]
\end{aligned}
$$

provided that

$$
\mathfrak{R}(\lambda+\mu)>1, \mathfrak{R}(\lambda)>0 \text {, and } \quad \mathfrak{R}(\mu)>0 .
$$

The conditions of validity of the above results follow easily from the conditions given by T. J. Osler[ 13, p. 664-665]).

Summation For mulae 2.5

$$
\begin{aligned}
& \sum_{n=-\infty}^{\infty}\left(\begin{array}{c}
\alpha \\
\eta+\boldsymbol{n}
\end{array}\right)\left[\frac{\Gamma(\mu)}{(\mu-\alpha+\eta+n)}{ }_{1} F_{2}\left(\mu ; \frac{1}{2}, \mu-\alpha+\eta+n ; \frac{a^{2} z}{4}\right) z^{1 / 2}\right. \\
+ & \left.\frac{a \Gamma\left(\mu+\frac{1}{2}\right)}{\left(\mu-\alpha+\eta+n+\frac{1}{2}\right)}{ }_{1} F_{2}\left(\mu+\frac{1}{2} ; \frac{1}{2}, \mu-\alpha+\eta+n+\frac{1}{2} ; \frac{a^{2} z}{4}\right)\right] \times\left[\frac{\Gamma(\lambda)}{(\lambda-\eta-n)}{ }_{1} F_{2}\left(\lambda ; \frac{1}{2}, \lambda-\eta-n ; \frac{b^{2} z}{4}\right) z^{1 / 2}\right. \\
& \left.+\frac{\left(b \Gamma\left(\lambda+\frac{1}{2}\right)\right.}{\left(\lambda-\eta-n+\frac{1}{2}\right)}{ }_{1} F_{2}\left(\lambda+\frac{1}{2} ; \frac{1}{2}, \lambda-\eta-n+\frac{1}{2} ; \frac{b^{2} z}{4}\right)\right] \\
= & {\left[\begin{array}{c}
\frac{\Gamma(\mu+\lambda-1)}{(\mu+\lambda-\alpha-1)}{ }_{1} F_{1}\left(\mu+\lambda-1 ; \frac{1}{2}, \mu+\lambda-\alpha-1 ; \frac{(a+b)^{2} z}{4}\right) \\
+\frac{(a+b) \Gamma\left(\mu+\lambda-\frac{1}{2}\right)}{\left(\mu+\lambda-\alpha-\frac{1}{2}\right)}{ }_{1} F_{2}\left(\mu+\lambda-\frac{1}{2} ; \frac{3}{2}, \mu+\lambda-\alpha-\frac{1}{2} ; \frac{(a+b)^{2} z}{4}\right) z^{1 / 2}
\end{array}\right] }
\end{aligned}
$$

where $\mathfrak{R}(\lambda+\mu)>1, \mathfrak{R}(\lambda)>0$, and $\mathfrak{R}(\mu)>0$.

The conditions of validity of the above results follow easily from the conditions given by T. J. Osler[ 13, p. 664-665]).

Proofs:

The results are obtained by assigning particular values to the functions $u(z)$ and $v(z)$ in the generalized Leibniz ru le (1.6). 
If we put $u(z)=z^{\mu-1} \cos a z \quad$ and $\quad v(z)=z^{\lambda-1} \cos b z$ in (1.6), then L.H.S. of (1.6) becomes

$$
D_{z}^{\alpha}[u(z) v(z)]=D_{z}^{\alpha}\left[\frac{1}{2} z^{\mu+\lambda-2}\{\cos (a+b) z+\cos (a-b) z\}\right]
$$

and using known result[4, p.189, eqn. (32)], we get

$$
\begin{gathered}
=\frac{1}{4} \frac{\Gamma(\mu+\lambda-1)}{\Gamma(\mu+\lambda-\alpha-1)}\left[{ }_{1} F_{1}(\mu+\lambda-1 ; \mu+\lambda-\alpha-1 ; i(a+b) z)+{ }_{1} F_{1}(\mu+\lambda-1 ; \mu+\lambda-\alpha-1 ;-i(a+b) z)\right. \\
\left.{ }_{1} F_{1}(\mu+\lambda-1 ; \mu+\lambda-\alpha-1 ; i(a-b) z)+{ }_{1} F_{1}(\mu+\lambda-1 ; \mu+\lambda-\alpha-1 ;-i(a-b) z)\right] z^{\mu+\lambda-\alpha-2}
\end{gathered}
$$

For R.H.S., we similarly have

$$
\begin{aligned}
& \sum_{n=-\infty}^{\infty}\left(\begin{array}{l}
\alpha \\
\eta+n
\end{array}\right) D_{z}^{\alpha-\eta-n}[u(z)] D_{z}^{\eta+n}[v(z)]=\sum_{n=-\infty}^{\infty}\left(\begin{array}{c}
\alpha \\
\eta+n
\end{array}\right) D_{z}^{\alpha-\eta-n}\left[z^{\mu-1} \cos a z\right] D_{z}^{\eta+n}\left[z^{\lambda-1} \cos b z\right], \text { (2.7) } \\
& D_{z}^{\alpha-\eta-n}\left[z^{\mu-1} \cos a z\right]=\frac{\Gamma(\mu)}{2 \Gamma(\mu-\alpha+\eta+n)}\left[{ }_{1} F_{1}(\mu ; \mu-\alpha+\eta+n ; i a z)+{ }_{1} F_{1}(\mu ; \mu-\alpha+\eta+n ;-i a z)\right] z^{\mu-\alpha+\eta+n-1} \\
& D_{z}^{\eta+n}\left[z^{\lambda-1} \cos b z\right]=\frac{\Gamma(\lambda)}{2 \Gamma(\lambda-\eta-n)}\left[{ }_{1} F_{1}(\lambda ; \lambda-\eta-n ; i b z)+{ }_{1} F_{1}(\lambda ; \lambda-\eta-n ;-i b z)\right] z^{\lambda-\eta-n-1}
\end{aligned}
$$

putting (2.6) and (2.7), in (1.6), we have the required result (2.1) after a little simplification:

Again, if we put $u(z)=z^{\mu-1} \sin a z \quad$ and $\quad v(z)=z^{\lambda-1} \cos b z$ in (1.6), proceed on similar lines as adopted in (2.1) and using known results [4, p.188, Eq. (21)], we obtained the required interesting formulae (2.2).

Next, If we take $u(z)=z^{\mu-1} L_{m}^{a}\left(\left(k_{1}+k_{2}\right) z\right) e^{-k_{1} z} \quad$ and $\quad v(z)=z^{\lambda-1} e^{-k_{2} z}$ in (1.6), proceed on similar lines as adopted in (2.1) and using known results [4, p.193, Eq. (51) and p.187, Eq. (14)], we arrive at the required interesting formu lae (2.3).

Further, on putting $u(z)=z^{\mu-1} \cos \left(a z^{1 / 2}\right) \quad$ and $\quad v(z)=z^{\lambda-1} \cos \left(b z^{1 / 2}\right)$ in (1.6), we easily obtained the formulae (2.4) after a little simplification on making use of similar lines of proof as adopted in (2.1) and using known results[4, p.190, Eq. (35)]:

Similarly, if we take $u(z)=z^{\mu-1} \exp \left(a z^{1 / 2}\right) \quad$ and $\quad v(z)=z^{\lambda-1} \exp \left(b z^{1 / 2}\right)$ in (1.6), we easily arrive at the required formulae (2.5) after a little simplification on making use of similar lines of proof as adopted in (2.1) and using known results[4, p.190, Eq. (35)]

\section{Special Cases}

In view of the large number of parameters involved in the summations of series established above, these summations of series are capable of yielding a number of known and new results. We record here only one special case for lack of space. For example:

If, we take $a=b=0 ; \mu=\mu-c ; \lambda=\lambda-d ; \alpha=\lambda-c-1$ and $\eta=\lambda-1$ in (2.1) and making use of the following well-known result on both the sides of the resulting result of (2.1) (cf., e.g., Erdély i et al.[4, p.185, Eqn. 13.1 (7)]):

$D_{z}^{-\alpha}\left\{z^{\lambda}\right\}=\frac{\Gamma(\lambda+1)}{\Gamma(\lambda+\alpha+1)} z^{\lambda+\alpha}(\mathfrak{R}(\alpha)>0 ; \mathfrak{R}(\lambda)>-1)$, We easily arrive at the well-known Dougall's formula[3, $\mathrm{p}$.

7, Eqn. 1.4(1)] after a little simplification.

\section{ACKNOWLEDGEMENTS}

The authors are thankful to the referee for the valuable comments and suggestions, which have led the paper to the present form.

\section{REFERENCES}

[1] M. Abramowitz, and I.A. Stegun, "Orthogonal Polynomials." Ch. 22 in Handbook of Mathematical Functions with Formulas, Graphs, and Mathematical Tables, 9th printing. New York: Dover, (1972), 771-802.

[2] M. P. Chen and H. M. Srivastava, Fractional calculus 
operators and their applications involving power functions and summation of series, Appl. Math. Comput. 81(1997), 287-304.

[3] A. Erdélyi, M. Magnus, F. Oberhettinger and F. G. Tricomi, Higher Transcendental Functions, Vol. I, McGraw-Hill Book Company, New York, Toronto, and London, (1953).

[4] A. Erdélyi, M. Magnus, F. Oberhettinger and F. G. Tricomi, Tables of Integral Transforms, Vol. II, McGraw-Hill Book Company, New York, Toronto, and London, (1954).

[5] L. Galué , S. L. Kalla and K. Nishimoto, Application of fractional calculus to infinite sums, J. Fractional Calculus 1 (1992), 17-21.

[6] L. Galué, Application of fractional calculus to infinite sums (II), J. Fractional Calculus 7(1995), 61-67.

[7] V. Kiryakova, Generalized Fractional calculus and Applications, Pitman Research Notes in Mathematics Series 301, Longman Scientific and Technical, Harlow, Essex (Johan Wiley and Sons, New York) (1994).

[8] R. Koekoek and R. F. Swarttouw, The Askey-Scheme of Hvbergeometric Orthogonal Polvnomials and its $q$ -Analogue. Delft, Netherlands: Technische Universiteit Delft, Faculty of Technical Mathematics and Informatics Report 98-17, pp. 47-49, 1998.

[9] K.S. Miller and B. Ross, An Introduction to the Fractional Calculus and Fractional Differential Equations, John Wiley and Sons, New York, Chichester, Brisbane, Toronto, and Singap ore (1993).
[10] K. Nishimoto, Fractional Calculus, Vols. I, II, III and IV, Descartes Press, Koriy ama (1984, 1987, 1989 and 1991).

[11] K. Nishimoto, An Essence of Nishimoto's Fractional Calculus (Calculus in the $21^{\text {st }}$ Century): Integrations and Differentiations of Arbitrary Order, Descartes Press, Koriy ama (1991).

[12] K.B. Oldham and J. Spanier, The Fractional Calculus, Academic Press, New York and London (1974).

[13] T. J. Osler, Leibniz rule for fractional derivatives gen eralized and an application to infinite series, SIAM J. Appl. Math., 18(1970), 658-574.

[14] S.G. Samko, A.A. Kilbas and O.I. Marichev, Fractional Integrals and Derivatives: Theory and Applications, Gordhan and Breach Science Publishers, Reading, Tokyo, Paris, Berlin and Langhorne (Pennsy lvania) (1993).

[15] H.M. Srivastava and P.W. Karlsson, Multiple Gaussian Hypergeometric Series, John Wiley and Sons, New York (1985).

[16] H. M. Srivastava and S. Owa, Univalent Functions, Fractional Calculus, and Their Applications, Halsted Press (Ellis Horwood Limited, Chichester), John Wiley and Sons, New York, Chichester, Brisbane, and Toronto (1989).

[17] Y. Watanabe, Notes on the generalized derivative of Riemann-Livouville and its applications to Leibniz's formula. I and II, Tohoku Math. J. 34 (1931), 8-27 and 28-41. 